\section{Biliary Reflux Due to Sphincter of Oddi Ablation: A New Pathogenetic Explanation for Long-Term Major Biliary Symptoms After Endoscopic- Sphincterotomy}

The short-term results and risks associated with endoscopic treatment for bile duct stones are well documented, but few prospective data concerning the long-term sequelae have been published. In a follow-up study, Escourrou reported major biliary symptoms in $10 \%$ of patients, $4-10 \%$ of whom develop stenosis or recurrent stones (1). Cotton recorded five patients who had stones, but no stenosis, after endoscopic sphincterotomy (ES), and who complained of recurrent symptoms in spite of two or more duct explorations. Most patients with recurrent biliary symptoms had air in the biliary tree and free reflux of barium into the bile duct (2). Duodenal biliary reflux after ES is frequently seen in barium studies. A $60-76 \%$ incidence of bacteriobilia has been reported at a second endoscopic retrograde cholangiopancreatography (ERCP) after ES $(1,3,4)$. The significance of these findings is not known, however, because the complications relating to the loss of sphincter of Oddi function are not well established. In two patients in our hospital who had previously undergone ES, and who had multiple episodes of cholangitis, the presence of food debris in the common bile duct clearly pointed to duodenal reflux in the pathogenesis.

Case 1: A 40-year-old man was admitted due to cholangitis. One year previously, he had undergone a cholecystectomy, followed by ES due to residual stones. He described having suffered selflimiting episodes of cholangitis eight and six months prior to the current hospital admission. Ultrasound and ERCP revealed an 11 $\mathrm{mm}$ common bile duct, without stenosis or lithiasis, and a $10-\mathrm{mm}$ open papilla. Ten days later, the patient was readmitted with cholangitis and hypotension. Laparotomy revealed an obstruction of the common bile duct by some dense material of vegetable origin (histological evidence). Blood cultures were positive for four of the usual gastrointestinal tract bacteria. Twenty-two months after the choledochoduodenostomy, there was no further evidence of biliary disturbances.

Case 2: Acute cholangitis was also the reason for admission in a 66-year-old woman, 18 months after she had undergone cholecystectomy and ES due to choledocholithiasis. Since then she had suffered two self-limiting episodes of cholangitis in which the ultrasound and ERCP studies did not show any residual stones or stenosis. On admission, some dense material of vegetable origin (histological evidence) was removed from the common bile duct during ERCP (Figure 1).

We suggest that the ablation of the sphincter of Oddi may induce free duodenal reflux into the common bile duct. Bacterial overgrowth and the presence of alimentary debris would be major factors in inducing cholestasis, cholangitis, and even relapse of stones in these patients. Aerobilia associated with a large main bile duct, free barium reflux, and a wide papilla may be predictive signs of duodenal biliary reflux capable of inducing recurrent cholangitis or major biliary symptoms. This is a new explanation for the conditions seen in a subgroup of patients who are usually classified under a well-known old syndrome, related to papillary stenosis or bad ductal clearance. Prospective studies of the late complications of ES should take into account the biliary reflux

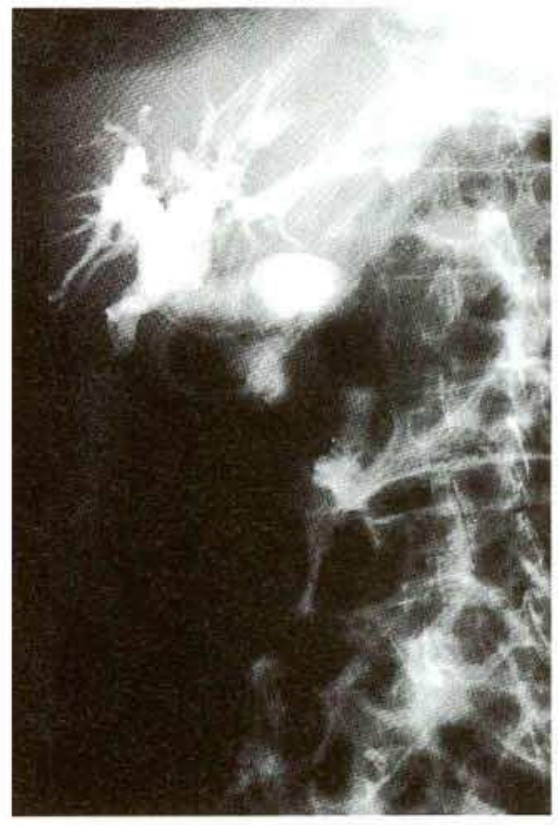

Figure 1: Endoscopic retrograde cholangiopancreatogram in a 66-year-old woman with acute cholangitis who had previously undergone endoscopic sphincterotomy. The histological analysis of the material retrieved from the common bile duct confirmed its vegetable origin

induced by complete ablation of the sphincter of Oddi as a potential cause of recurrent self-limiting cholangitis.

J. M. Bordas, I. Elizalde, J. Llach, F. Mondelo, R. Bataller, J. Terès Digestive Endoscopy Section, Hospital Clinic, University of

Barcelona, Barcelona, Spain

\section{References}

1. Escourrou J, Cordova JA, Lazorthes F, et al. Early and late complications after endoscopic sphincterotomy for bilary lithiasis, with and without the gallbladder "in situ". Gut 1984; 25: $598-602$.

2. Cotton PB. Endoscopic management of bile duct stones (apples and oranges). Gut 1984; 25: 587-97.

3. Hawes RH, Cotton PB, Vallon AG. Follow-up 6 to 11 years after duodenoscopic sphincterotomy for stones in patients with prior cholecystectomy. Gastroenterology 1990; 98: 1008-12.

4. Ikeda S, Tanaka M, Matsumoto S, Yoshimoto H, Itoh $\mathrm{H}$. Endoscopic sphincterotomy: long-term results in 408 patients with complete follow-up. Endoscopy 1988; 20: 13-7.

\section{Corresponding Author}

J. M. Bordas, M. D.

Seccio d'Endoscopia Digestiva

Hospital Clinic I Provincial

Villarroel 170

08036 Barcelona

Spain

Fax: +34-3-2275454 